\title{
Cryptococcose disséminée chez une greffée rénale de longue date
}

\author{
Kelli Li MD, Abid Khan MD, Sharmistha Mishra MD PhD MSc, Alice Zhabokritsky MD MSc
}

- Citation : CMAJ 2021 February 8;193:E211-4. doi : 10.1503/cmaj.200825-f

Voir la version anglaise de l'article ici : www.cmaj.ca/lookup/doi/10.1503/cmaj.200825

$\mathbf{U}$ ne femme de 55 ans a été hospitalisée pour une pneumonie. Elle avait subi une greffe rénale 14 ans plus tôt pour une maladie polykystique des reins, après quoi elle avait présenté une néphropathie chronique d'allogreffe caractérisée par un taux de créatinine de référence de $250 \mu \mathrm{mol} / \mathrm{L}$ (plage normale 42-102 $\mu \mathrm{mol} / \mathrm{L}$ ). La patiente ne présentait aucune autre comorbidité. Elle prenait de la prednisone $5 \mathrm{mg}$ DIE, de la cyclosporine A $50 \mathrm{mg}$ BID (taux moyen de cyclosporine 2 heures postdose 500-700 ng/L), et du mycophénolate mofétil (MMF) $500 \mathrm{mg}$ BID.

Trois semaines avant son hospitalisation, la patiente avait séjourné dans un chalet à l'île-du-Prince-Édouard, mais n'avait pas fréquenté de ferme et n'avait pas été exposée à des animaux ou à des fientes d'oiseaux. La semaine suivante, elle avait eu de la diarrhée non sanglante. L'équipe de transplantation qui la suivait à l'externe avait alors proposé de stopper le MMF en raison de son association connue avec la diarrhée. Puis, 10 jours avant l'admission, elle a présenté de légères céphalées et de la léthargie, accompagnées d'une toux sèche et de nausées. Enfin, la veille de son arrivée à l'hôpital, elle a développé de la fièvre. Selon l'examen réalisé au service des urgences, la patiente était fébrile $\left(38,2^{\circ} \mathrm{C}\right)$, hypertendue (pression artérielle 186/99 $\mathrm{mm} \mathrm{Hg}$ ) et tachycarde (104 battements/minute). Sa saturation périphérique en oxygène était de $97 \%$ à l'air ambiant. Les premières analyses sanguines ont donné les résultats suivants : numération leucocytaire, $8,4 \times 10^{9} / \mathrm{L}$ (plage normale $4-11 \times 10^{9} / \mathrm{L}$ ); créatinine, $358 \mu \mathrm{mol} / \mathrm{L}$ (plage normale $42-102 \mu \mathrm{mol} / \mathrm{L}$ ); potassium, $4,6 \mathrm{mmol} / \mathrm{L}$ (plage normale $3,5-5 \mathrm{mmol} / \mathrm{L}$ ); et urée, $32,5 \mathrm{mmol} / \mathrm{L}$ (plage normale $3-7 \mathrm{mmol} / \mathrm{L}$ ). Des examens d'imagerie thoracique par radiographie et tomodensitométrie ont révélé la présence de consolidations à la base des 2 poumons, accompagnées d'un œdème pulmonaire (figure 1). Des échantillons sanguins ont été prélevés pour une hémoculture, et la patiente a commencé un traitement de pipéracilline/ tazobactam avec ajustement de la dose selon la fonction rénale pour une pneumonie présumée. On a amorcé une hémodialyse pour corriger l'hypervolémie et cessé la cyclosporine A et le MMF.

\section{POINTS CLÉS}

- Les patients qui subissent une greffe d'organe plein suivent un traitement immunosuppresseur à long terme qui accroît leur risque de présenter une cryptococcose disséminée.

- Les caractères épidémiologiques des cryptococcoses disséminées ont changé dans les 10 dernières années; l'incidence de ces infections a augmenté chez les patients non infectés par le VIH.

- Une méningoencéphalite cryptococcique devrait être soupçonnée chez les patients immunodéficients qui présentent de la fièvre et des symptômes neurologiques généraux.

- Une cryptococcose invasive et une infection concomitante par le cytomégalovirus pourraient être associées à un risque accru de morbidité et de décès.

Dans les 48 heures suivantes, la patiente est restée fébrile et hypertendue, et son état de conscience s'est détérioré. Trois jours après son hospitalisation, elle présentait un léger méningisme, des spasmes myocloniques intermittents aux membres inférieurs, ainsi qu'une faiblesse pyramidale symétrique affectant ses bras et ses jambes. Les muscles proximaux de ses bras et de ses jambes étaient légèrement plus faibles (4- sur 5 selon l'échelle du Conseil de recherches médicales) que les muscles distaux (4 sur 5 ), mais leur masse et leur tonus étaient relativement normaux. Ses réflexes tendineux profonds bilatéraux étaient bons, et ses réponses plantaires, présentes. Les tests de coordination et les tests sensoriels n'ont montré aucune anomalie importante.

Une imagerie par résonance magnétique (IRM) cérébrale d'urgence a montré la présence de foyers anormaux semblables à des bulles hyperintenses en pondération $T_{2}$ et sans restriction de la diffusion dans les noyaux gris centraux bilatéraux, ainsi que d'un léger œdème vasogénique (figure 2). L'analyse du liquide céphalorachidien (LCR) prélevé par ponction lombaire a révélé une protéinorachie de $0,9 \mathrm{~g} / \mathrm{L}$ (plage normale $0,15-0,45 \mathrm{~g} / \mathrm{L}$ ), une proportion glycorachie-glycémie de $48 \%$ (valeur normale $>60 \%$ ) et une numération leucocytaire de $6 \times 10^{6} / \mathrm{L}$ (plage normale $0-5 \times 10^{6} / \mathrm{L}$ ) [39\% de 

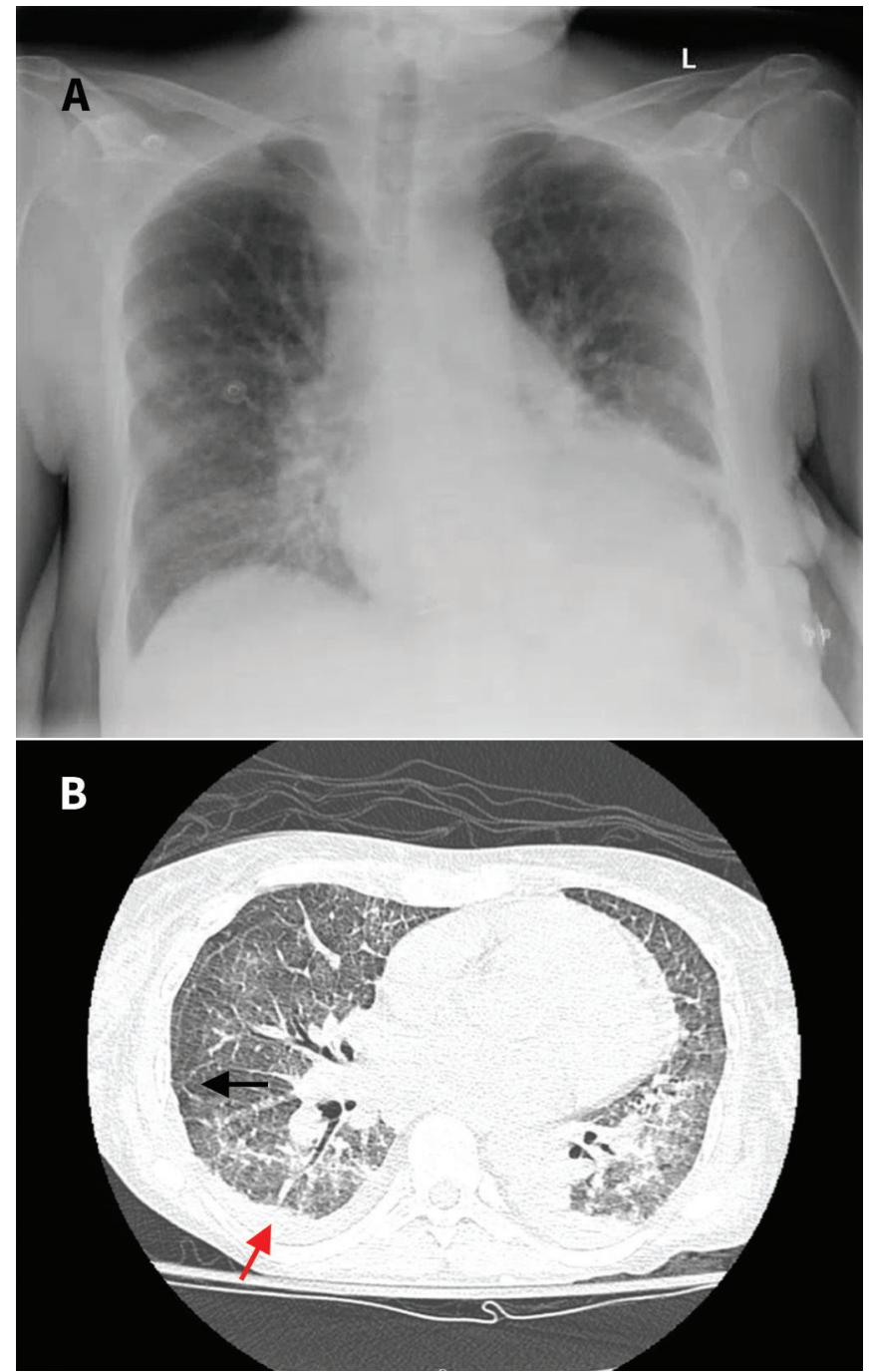

Figure 1 : A) Radiographie thoracique montrant de petites consolidations à la base des deux poumons chez une femme de 55 ans. On y voit également d'importantes lésions interstitielles diffuses. B) Tomodensitométrie thoracique sans produit de contraste montrant des consolidations - principalement à la base des deux poumons -, ainsi qu'un épaississement septal interlobulaire (flèche noire) et de petites effusions pleurales bilatérales (flèche rouge). Ces images indiquaient la présence d'un processus infectieux ou inflammatoire accompagné d'un œdème pulmonaire.

neutrophiles, $25 \%$ de lymphocytes et $34 \%$ de monocytes]. La pression d'ouverture était de $38 \mathrm{~cm} \mathrm{H}_{2} \mathrm{O}$ (valeur normale $<20 \mathrm{~cm} \mathrm{H}_{2} \mathrm{O}$ ).

Étant donné la présence de fièvre, l'altération de l'état de conscience, les lésions des noyaux gris centraux et la pression d'ouverture élevée, nous avons présumé que cette patiente immunodéficiente présentait une méningite cryptococcique et avons amorcé un traitement empirique $(4 \mathrm{mg} / \mathrm{kg} / \mathrm{j}$ d'amphotéricine $B$ liposomale IV et $1500 \mathrm{mg}$ de flucytosine PO après la dialyse). Peu après, nous avons reçu les résultats des tests sérologiques, qui faisaient état de la présence de l'antigène cryptococcique dans le sérum et le LCR, à un titre de > 1:1024 (Cryptococcal Antigen Latex Agglutination System de Meridian Bioscience). Quatre jours après son admission à l'hôpital, la
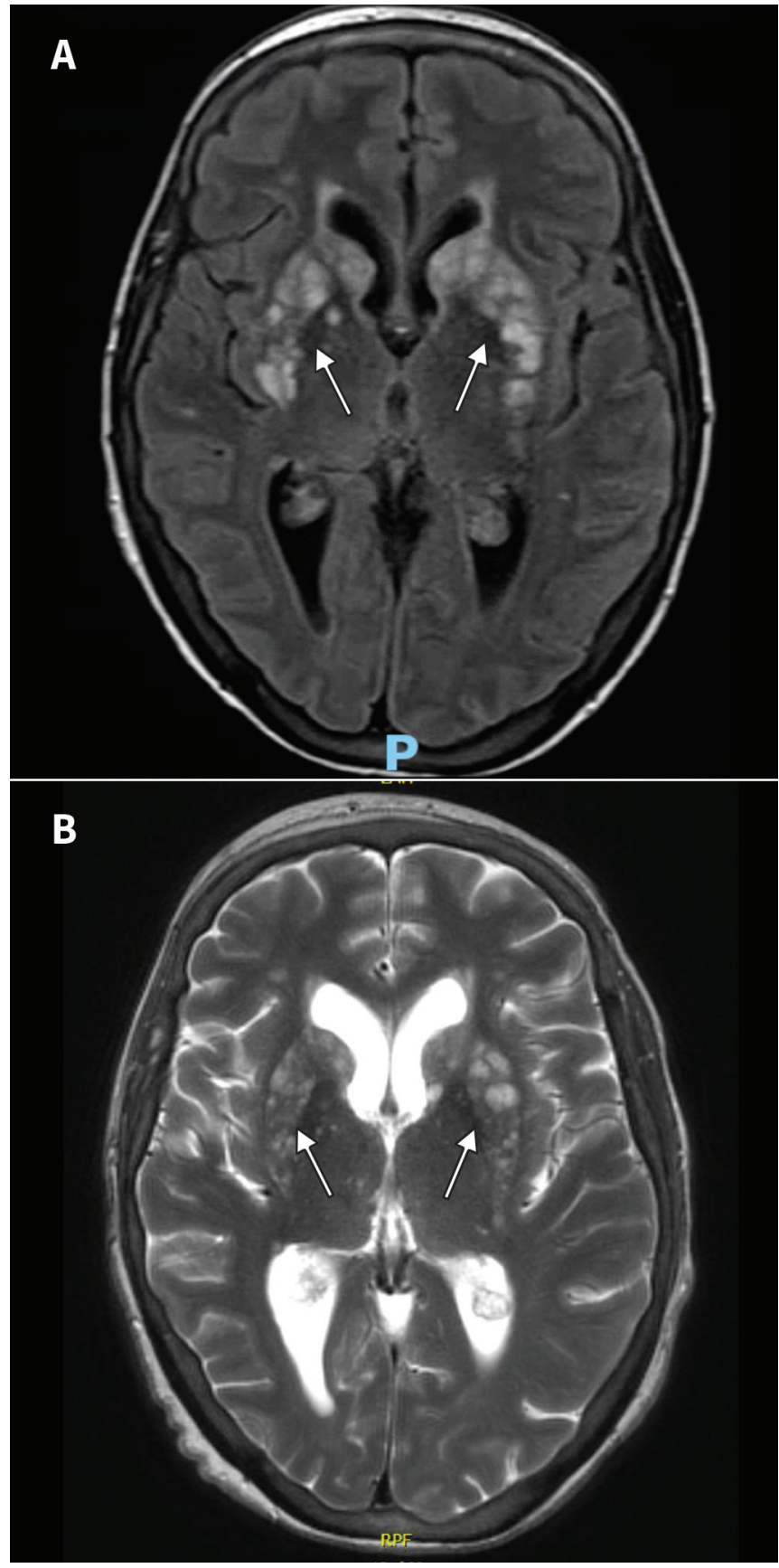

Figure 2 : Imagerie par résonance magnétique cérébrale axiale sans gadolinium (en raison de la dysfonction rénale) montrant des foyers anormaux semblables à des bulles dans les noyaux gris centraux bilatéraux (flèches). Ces foyers produisaient un signal hyperintense A) en séquence FLAIR (fluid-attenuated inversion recovery) avec pondération $T_{2}$; et $\mathrm{B}$ ) en pondération $T_{2}$. Ces images indiquaient la présence de pseudokystes gélatineux ou d'une cryptococcose. On y observe également un léger œdème vasogénique sans effet de masse important.

patiente a présenté une décompensation respiratoire progressive nécessitant une intubation. Au cinquième jour, l'hémoculture a donné des résultats positifs, et l'agent pathogène Cryptococcus neoformans a été identifié au moyen d'une spectrométrie de masse par désorption-ionisation laser assistée par matrice. Des cultures de LCR et de sécrétions respiratoires 
subséquentes ont également fait état d'une forte croissance de C. neoformans. Les cultures bactériennes du LCR, le dépistage par frottis de bacilles acido-résistants, la recherche des virus de l'herpès et du Nil occidental par test d'amplification en chaîne par polymérase (PCR), et le dépistage de la syphilis ont quant à eux donné des résultats négatifs. La détection du cytomégalovirus dans le sang par PCR était positive $(3,6 \times 104$ copies $/ \mathrm{mL})$, et le dépistage du VIH, négatif.

La pression d'ouverture demeurant élevée chez la patiente, des ponctions lombaires ont été réalisées chaque jour. Leur but était de réduire cette pression de plus de $50 \%$, de façon à ce qu'elle soit $<15 \mathrm{~cm} \mathrm{H}_{2} \mathrm{O}$. Un drain lombaire a été installé au jour 6. Le lendemain, la prednisone a été cessée au profit d'un sevrage à la dexaméthasone pour traiter l'œdème vasogénique. Les cultures du LCR ont donné des résultats positifs jusqu'au jour 9. On a retiré le drain lombaire au jour 13, une fois la pression d'ouverture redevenue normale. À la neuvième semaine, on a commencé à administrer à la patiente un traitement de consolidation au fluconazole, à raison de $800 \mathrm{mg}$ PO après la dialyse, mais son rétablissement neurologique a été minimal. Elle a eu besoin de dialyse durant tout son séjour à l'hôpital. Après des épisodes récurrents de sepsis nosocomial et de sepsis lié aux cathéters, il a été décidé que la patiente, alors hospitalisée depuis presque 6 mois, recevrait dorénavant des soins de confort.

\section{Discussion}

La cryptococcose est la troisième infection fongique invasive en importance, après la candidose et l'aspergillose, chez les personnes ayant subi une transplantation. En effet, elle représente $8 \%$ des cas d'infections fongiques chez cette population ${ }^{1}$. Selon un examen de rapports publiés entre 1998 et 2001, sa forme disséminée toucherait 0,3\%-5,3\% des personnes greffées ${ }^{2}$. Comparativement aux autres infections fongiques invasives, la cryptococcose prend plus de temps à se manifester après la chirurgie ( 575 jours pour la cryptococcose, c. 103 jours pour la candidose invasive et 184 jours pour l'aspergillose).

Les caractères épidémiologiques de la cryptococcose invasive semblent changer. Au Canada, on estime que son incidence est de 63 cas pour 100000 personnes ${ }^{4}$. Par ailleurs, avant 2010, la cryptococcose touchait surtout les patients atteints du $\mathrm{VIH}$, tandis que maintenant, plus de $90 \%$ des cas surviennent chez des personnes séronégatives ${ }^{5}$. S'il en est ainsi, c'est entre autres parce que les traitements antirétroviraux sont amorcés plus tôt chez les personnes infectées par le VIH, mais aussi parce que le nombre de personnes greffées prenant des immunosuppresseurs a augmenté.

Cryptococcus neoformas et Cryptococcus gattii sont les 2 cryptocoques les plus fréquemment associés à l'infection chez les humains. C. neoformans est très répandu dans l'environnement : on le trouve notamment dans le sol, le bois en décomposition et les fientes d'oiseaux ${ }^{1}$. La patiente l'a probablement contracté en inhalant des spores fongiques, qui constituent son principal mode de transmission. Générale- ment signalé chez les personnes immunodéficientes, il est la principale cause de méningite chez les personnes se trouvant à un stade avancé de l'infection par le $\mathrm{VIH}^{1}$. Quant à C. gattii, il s'attaque surtout aux personnes immunocompétentes ${ }^{1}$. On le trouve habituellement dans les régions tropicales et subtropicales, quoique des éclosions ont été signalées en Colombie-Britannique ${ }^{1,4}$.

Une méningoencéphalite cryptococcique devrait être soupçonnée chez les personnes immunodéficientes qui présentent des symptômes neurologiques généraux et de la fièvre, comme notre patiente. Plus de $75 \%$ des patients font état de céphalées insidieuses, et moins de $25 \%$ présentent des signes de méningismes ${ }^{6}$. Les autres symptômes courants comprennent une altération de l'état de conscience (environ $50 \%$ des cas), des troubles visuels (environ $20 \%$ des cas) et des convulsions ou des déficits neurologiques ( $<10 \%$ des cas), qui pourraient indiquer la présence d'une lésion occupant de l'espace, comme un cryptococcome ${ }^{6}$. Les examens radiographiques pourraient révéler la présence d'une méningoencéphalite, de granulomes (cryptococcomes) ou de pseudokystes gélatineux, souvent dans les noyaux gris centraux ${ }^{7}$. Le diagnostic différentiel des lésions des noyaux gris centraux chez les patients immunodéficients comprend la cryptococcose, la toxoplasmose cérébrale, l'encéphalite virale et les affections malignes ${ }^{7}$.

Les traitements immunosuppresseurs, en particulier ceux qui modulent l'immunité cellulaire, influent sur les risques et les issues des cryptococcoses systémiques. En général, les anticorps induisant la déplétion des lymphocytes $\mathrm{T}$ sont associés au plus grand risque de cryptococcose après une transplantation ${ }^{8}$. Les inhibiteurs de la calcineurine, comme la cyclosporine $\mathrm{A}$ et le tacrolimus, ont été associés à une diminution du taux de mortalité à 90 jours et à une augmentation de la survie à long terme, comparativement à l'azathioprine et au MMF. Les traitements par ces inhibiteurs ont également été associés à moins d'infections du système nerveux central que ceux à l'azathioprine ou au MMF'. Une augmentation du risque d'infection à $C$. neoformans a été signalée chez les patients prenant des stéroïdes à long terme pour des troubles auto-immuns, mais peu de données montrent l'existence d'un lien entre la posologie du traitement aux stéroïdes et le risque de cryptococcose chez les personnes ayant subi une transplantation ${ }^{10}$.

Notre patiente est venue consulter pour une grave infection à $C$. neoformans 14 ans après sa greffe rénale. Nous avons aussi recensé un cas d'infections urinaires récurrentes 13 ans après une transplantation; ces infections avaient été associées à un cryptococcome dans le rein greffé ${ }^{11}$. Dans ce dernier cas, la patiente avait reçu des stéroïdes de façon intermittente et avait changé son traitement immunosuppresseur 3 ans plus tôt en raison d'un déclin rapide de sa fonction rénale. Contrairement à elle, notre patiente était stable sur le plan clinique et prenait $5 \mathrm{mg}$ de prednisone par jour depuis plus de 10 ans, en plus de la cyclosporine $A$ et du MMF. Tout de suite après sa greffe, elle avait présenté un rejet aigu, qui avait été traité à la globuline antithymocyte; elle n'avait eu aucune autre complication qui aurait nécessité 
un traitement induisant une déplétion des lymphocytes T ou l'administration intermittente de stéroïdes.

Notre patiente présentait plusieurs facteurs augmentant le risque d'issues indésirables, comme un titre élevé d'antigène cryptococcique dans le LCR, une faible proportion glycorachieglycémie, une pression intracrânienne élevée et une altération de l'état de conscience ${ }^{6}$. Par ailleurs, elle avait une infection concomitante par le cytomégalovirus (CMV), qui a été associée à un taux de mortalité accru chez les patients sidatiques ${ }^{12}$. Les conséquences d'une infection par le CMV et des infections fongiques opportunistes concomitantes en ce qui a trait à la morbidité et au décès sont méconnues chez les personnes ayant subi une transplantation. De récentes données ex vivo de patients greffés ont montré que l'infection par le CMV atténue la réponse des cytokines aux infections comorbides, ce qui pourrait expliquer l'effet immunomodulateur du CMV chez cette population ${ }^{13}$.

\section{Conclusion}

Nous signalons un cas de cryptococcose avec méningoencéphalite, pneumonie et infection concomitante par le CMV chez une patiente ayant subi une greffe rénale plusieurs années auparavant et dont le traitement immunosuppresseur, qui comprenait la prise quotidienne d'une faible dose de prednisone, était stable. Une infection fongique invasive, comme une cryptococcose, devrait être soupçonnée chez les personnes qui font de la fièvre et qui présentent des symptômes neurologiques généraux, afin de favoriser un diagnostic et une prise en charge précoces.

\section{Références}

1. Pappas PG, Alexander BD, Andes DR, et al. Invasive fungal infections among organ transplant recipients: results of the transplant-associated infection surveillance network (TRANSNET). Clin Infect Dis 2010;50:1101-11.

2. Husain S, Wagener MM, Singh N. Cryptococcus neoformans infection in organ transplant recipients: variables influencing clinical characteristics and outcome. Emerg Infect Dis 2001;7:375-81.

3. Maziarz EK, Perfect JR. Cryptococcosis. Infect Dis Clin North Am 2016;30: 179-206.

4. Dufresne SF, Cole DC, Denning DW, et al. Serious fungal infections in Canada. Eur J Clin Microbiol Infect Dis 2017;36:987-92.

5. Patel V, Desjardins M, Cowan J. Shift in epidemiology of cryptococcal infections in Ottawa with high mortality in non-HIV immunocompromised patients. J Fungi (Basel) 2019;5:104.

6. Zunt JR, Baldwin KJ. Chronic and subacute meningitis. Continuum (Minneap Minn) 2012;18:1290-318.

7. Van Cauter S, Severino M, Ammendola R, et al. Bilateral lesions of the basal ganglia and thalami (central grey matter): pictorial review. Neuroradiology 2020;62:1565-605.
8. Silveira FP, Husain S, Kwak EJ, et al. Cryptococcosis in liver and kidney transplant recipients receiving anti-thymocyte globulin or alemtuzumab. Transpl Infect Dis 2007;9:22-7.

9. Singh N, Alexander BD, Lortholary O, et al. Cryptococcal Collaborative Transplant Study Group. Cryptococcus neoformans in organ transplant recipients: impact of calcineurin-inhibitor agents on mortality. J Infect Dis 2007;195: 756-64.

10. Kerr C, Stack WA, Sadlier C, et al. Disseminated cryptococcal infection initially presenting as cryptococcal cellulitis in an HIV-negative patient on long-term steroids. BMJ Case Rep 2018;11:e227249.

11. Muranda AZ, Greeff L, Sathekge MM, et al. Cryptococcoma of a transplanted kidney in a patient presenting with recurrent urinary tract infection: a case report. BMC Nephrol 2018;19:94.

12. Skipper C, Schleiss MR, Bangdiwala AS, et al. Cytomegalovirus viremia associated with increased mortality in cryptococcal meningitis in Sub-Saharan Africa. Clin Infect Dis 2020;71:525-31.

13. L'Huillier AG, Ferreira VH, Ku T, et al. Improving our mechanistic understanding of the indirect effects of CMV infection in transplant recipients. Am J Transplant 2019;19:2495-504.

Intérêts concurrents : Aucun déclaré.

Cet article a été révisé par des pairs.

Les auteurs ont obtenu le consentement de la patiente.

Affiliations : Divisions de médecine interne générale (Li), de néphrologie (Khan) et d'infectiologie (Mishra, Zhabokritsky), Département de médecine, Faculté de médecine, Université de Toronto; Division d'infectiologie (Mishra), Service de médecine, Hôpital St. Michael, Unity Health Toronto, Toronto, Ont.

Collaborateurs : Kelli Li a conçu le travail et rédigé le manuscrit. Tous les auteurs ont révisé de façon critique le contenu intellectuel important du manuscrit; ils ont donné leur approbation finale pour la version destinée à être publiée et assument l'entière responsabilité de tous les aspects du travail.

Propriété intellectuelle du contenu : Il s'agit d'un article en libre accès distribué conformément aux modalités de la licence Creative Commons Attribution (CC BY-NC-ND 4.0), qui permet l'utilisation, la diffusion et la reproduction dans tout médium à la condition que la publication originale soit adéquatement citée, que l'utilisation se fasse à des fins non commerciales (c.-à-d., recherche ou éducation) et qu'aucune modification ni adaptation n'y soit apportée. Voir : https://creativecommons.org/licenses/by-nc-nd/4.0/deed.fr.

Financement : La publication en accès ouvert de ce travail a été financée par le Programme de subventions Fondation (FN 13455) des Instituts de recherche en santé du Canada.

Remerciements : Les auteurs remercient le $D^{r}$ Raphael Schneider (Service de neurologie, Hôpital St. Michael, Toronto [Ontario]) pour son point de vue précieux sur les aspects neurologiques de cette étude de cas.

Correspondance : Alice Zhabokritsky, Alice.Zhabokritsky@one-mail.on.ca 